\title{
Dynamical Symmetries, Super-coherent States and Noncommutative Structures: Categorical and Geometrical Quantization Analysis
}

\author{
Diego Julio Cirilo-Lombardo ${ }^{1,2}$
}

(C) Springer (India) Private Ltd., part of Springer Nature 2018

\begin{abstract}
The relation between fundamental spacetime structures and dynamical symmetries are treated beyond the geometrical and topological viewpoint. To this end analyze, taking into account the concept of categories and quasi hamiltonian structures, a recent research (CiriloLombardo and Arbuzov in Int J Geom Methods Mod Phys 15(01):1850005, 2017) where one linear and one quadratic in curvature models were constructed and where a dynamical breaking of the $S O(4,2)$ group symmetry arises. We explain there how and why coherent states of the Klauder-Perelomov type are defined for both cases taking into account the coset geometry and some hints on the possibility to extend they to the categorical (functorial) status are given. The new spontaneous compactification mechanism that was defined in the subspace invariant under the stability subgroup is commented in the context of future developments as the main tool for the treatment of the internal symmetries, as the electroweak in the Standard Model (SM). The physical implications of the symmetry rupture as the introduction of a noncommutative structure in the context of non-linear realizations and direct gauging are analyzed and briefly discussed in this new theoretical framework.
\end{abstract}

Keywords Coherent states - Dynamical symmetries - Geometrical quatization - UFT · Categories · Group manifolds

\section{Contents}

Introduction

Noncommutative Structures

$\triangle$ Diego Julio Cirilo-Lombardo diego777jcl@gmail.com

1 Consejo Nacional de Investigaciones Cientificas y Tecnicas (CONICET), Universidad de Buenos Aires, National Institute of Plasma Physics (INFIP), Facultad de Ciencias Exactas y Naturales, Ciudad Universitaria, 1428 Buenos Aires, Argentina

2 Bogoliubov Laboratory of Theoretical Physics, Joint Institute for Nuclear Research, Dubna, Russian Federation 141980 
Gauge Theories of Gravity .

Cartan Forms, Pullbacks and Quantization

Cosets and Number of Fields

Higher Structures in Field Theory . .

Coset Coherent States and Quasihamiltonian Structures

Invariant $S O(2,4)$ Action and Breakdown Mechanism

Linear in $R^{A B}$

$A=m$ and $B=\lambda$

$A=m(x)$ and $B=\lambda(x)$ : Spontaneous Subspace

Supergravity as a Gauge Theory and Topological QFT

Quadratic in $R^{A B}$

Maxwell Equations and the Electromagnetic Field

Equations of Motion in Components and Symmetries

Nonlinear Realizations Viewpoint

Symplectic Structures, Poisson Manifolds and Noncommutativity

Generalization of Rothstein's Theorems Even Supersymplectic Supermanifols

Tangent Space and Even Supermanifold Structure

The Geometrical Reduction and Even Symplectic Super-Metrics

Example: Volkov-Pashnev Metric

Discussion

Appendix I: Symmetry Breaking Mechanism: The $S O(4,2)$ Case

A. General Features

Goldstone Fields and Symmetries

References

\section{Introduction}

As we recently have been discussed [73], studies of higher-dimension theories that involve (spontaneously) broken symmetries and noncommutativity in the quantum case are motivated by searches for a unified theory and consequently by a consistent theory of quantum gravity. Dimensional reduction of such theories is not unique and becomes extremely involved when gravity is included. We believe that the guiding principles for the reduction are provided by the observed (or desirable) physical field content and by the group theoretical structure itself. It is possible, however, to include more fundamental structures (categories) that allow a more natural way of describing all the properties of spacetime that interest us. In the other hand, symplectic geometry grew out of the theoretical study of classical and quantum mechanics. At first it was thought that it differs considerably from Riemannian geometry, which developed from the study of curves and surfaces in three dimensional Euclidean space, and went on to provide the language in which General Relativity is studied. This fact was understandable given that symplectic geometry started from the study of phase spaces for mechanical systems but, with the subsequent seminal works of Cartan that introduce the symplectic structure into the geometry of the spacetime calculus, that thinking changed radically due the introduction of the concept of categories and functors. In this paper we review and give some new results our recent research introducing some new ideas and results both, from the physical and mathematical viewpoint.

\section{Noncommutative Structures}

From the technical point of view, we have to extend physical fields into an extra (internal) space with preserving the general noncommutative quantum structure. However from the point of view of only group manifolds, the development of a mechanism that permit us to display the set of physical fields in interaction with the corresponding four dimensional world 
implies that some of the original symmetries of the higher-dimension manifold have been broken. There exist many theoretical attempts to realize the above ideas such as string and brane theories but none of them can be treated as the final answer: formulation of such theories contain serious problems that are still non solved. In spite of the fact that in these theories the solution seems to include a non-commutative structure [1,2], the concrete implementation of these symmetries in a substructure of any (super) manifold seems to be very complicated from the technical and geometrical viewpoints. However the possible answer to this question as for the problems of the geometrical quantization procedures which include a categorization mechanism. A posibility is given in Section VI where we explain the generalized Rothstein theorem presented by us before and may include naturally the desired categorization.

\section{Gauge Theories of Gravity}

However if well there exist another way to attack the unification problem that is in the context of gauge theories of gravity [3-5], the quantum picture is still not clear. The main problem is to conciliate the gauge theories, the breaking of symmetry and the mechanism of quantization in a fiber bundle structure. We will not go into details of each proposed theory of gravitation here, only the possibility of implementing a consistent geometric quantization scheme. As is well known the first model of gauge gravitation theory was suggested by Utiyama [6] in 1956 generalizaing the original $S U(2)$ gauge model of Yang and Mills to an arbitrary symmetry Lie group he met the problem of treating general covariant transformations and a pseudoRiemannian metric which had no partner in the Yang-Mills gauge theory see also [3,4,7-11] and references therein. Since the Poincaré group comes from the Wigner-Inonu contraction of de Sitter groups $S O(2,3)$ and $S O(1,4)$ and it is a subgroup of the conformal group, gauge theories on fibre bundles with these structure groups were also considered [12-18] . Because these fibre bundles fail to be natural, the lift of the group Diff(X) of diffeomorphisms of the fiber onto the base should be defined $[19,20]$. However, these gauging approaches contain the problem with a non-linear (translation) summand of an affine connection being a soldering form, but neither a frame (vierbein) field nor a tetrad field. Thus the latter doesn't have the status of a gauge field [21-23]. At the same time, a gauge theory in the case of spontaneous symmetry breaking also contains classical Higgs fields, besides the gauge and matter ones [24-32]. Therefore, basing on the mathematical definition of a pseudoRiemannian metric, some authors formulated gravitation theory as a gauge theory with a reduced Lorentz structure where a metric gravitational field is treated as a Higgs field [3337]. Consequently all the above attempts to implement a clean geometrical quantization procedure fail justifying the possibility of more fundamental algebro-geometric structures at the level of the base differentiable manifold.

\section{Cartan Forms, Pullbacks and Quantization}

The most satisfactory answer to the formulation of gravity as a gauge theory was developed in the pure geometrical context in the works of Volkov et al. [38-41]; in the context of supergravity by Arnowitt and Pran Nath [42]; and finally by Mansouri [43] who was able to solve some of the problems listed before by means of a principal fiber bundle imposing a condition of orthogonality of the generators of the fiber and base manifold. Such conditions that break the symmetry of the original group are implemented by means of a particular choice of the metric tensor. This approach was implemented in a supergroup structure obtaining a gauge theory of supergravity. Note that the underlying geometry must be reductive (in the Cartan sense) or weakly reductive in the case of supergravity. In these cases a geometrical 
quantization procedure can be incorporated because there ias a correct supergroup structure with a Cartan weakly reductive geometry.

\section{Cosets and Number of Fields}

As always, even the problem to determine which fields transform as gauge fields and which not, as well as which fields are physical ones and which are redundant, nonetheless remains. Also the relation between the coset factorization (as in the case of the non-linear realization approach [52-54]) and the specific breaking of the symmetry in the pure topological theories of grand unification (GUT) is still unclear.

\section{Higher Structures in Field Theory}

Gerbes appear in descriptions of the classical fields on manifolds and their boundaries by Dan Freed. There have to be links via "twisted K-theory" with Mickelsson's work on QFT [72] (and references therein), anomalies and gerbes. The latter involves (twisted) projective representations (as opposed to linear representations) of the group of classical symmetries, on a Hilbert space of quantum states. Such "anomalies" can often be expressed in terms of Dixmier-Douady classes (in the integer-valued third cohomology group) or in terms of gerbes, or via twisted K-theory. I think that it would be nice to understand this point better.

\section{Coset Coherent States and Quasihamiltonian Structures}

Let us remind the definition of coset coherent states

$$
H_{0}=\left\{g \in G \mid \mathcal{U}(g) V_{0}=V_{0}\right\} \subset G .
$$

Consequently the orbit is isomorphic to the coset, e.g.

$$
\mathcal{O}\left(V_{0}\right) \simeq G / H_{0}
$$

Analogously, if we remit to the operators, e.g.

$$
\left|V_{0}\right\rangle\left\langle V_{0}\right| \equiv \rho_{0}
$$

then the orbit

$$
\mathcal{O}\left(V_{0}\right) \simeq G / H
$$

with

$$
\begin{aligned}
H & =\left\{g \in G \mid \mathcal{U}(g) V_{0}=\theta V_{0}\right\} \\
& =\left\{g \in G \mid \mathcal{U}(g) \rho_{0} \mathcal{U}^{\dagger}(g)=\rho_{0}\right\} \subset G .
\end{aligned}
$$

The orbits are identified with coset spaces of $G$ with respect to the corresponding stability subgroups $H_{0}$ and $H$ being the vectors $V_{0}$ in the second case defined within a phase. From the quantum viewpoint $\left|V_{0}\right\rangle \in \mathcal{H}$ (the Hilbert space) and $\rho_{0} \in \mathcal{F}$ (the Fock space) are $V_{0}$ normalized fiducial vectors (an embedded unit sphere in $\mathcal{H}$ ).

In the case of Hamiltonian and quasihamiltonian structures the typical case can be exemplified as follows

$$
\begin{array}{r}
G(\Sigma) \text { maps }: \Sigma \rightarrow G \\
\curvearrowright \\
\Omega^{1}(\Sigma) \otimes g
\end{array}
$$


now

$$
g: \Sigma \rightarrow G
$$

and we have a connection such is invariant under

$$
A \rightarrow g^{-1} A g+g^{-1} d g
$$

is the action with A hamiltonian? We define $t: \Sigma \rightarrow g$, then:

$$
H_{t}(A)=\int_{\Sigma}\left\langle t, F_{A}\right\rangle+\int_{\partial \Sigma}\langle t, A\rangle
$$

where $F_{A}=d A+A \wedge A$ and looking at the Poisson bracket between 2 actions:

$$
\left\{H_{t_{1}}, H_{t_{2}}\right\}=H_{\left[t_{1}, t_{2}\right]}+\int_{\partial \Sigma}\left\langle t_{1}, d t_{2}\right\rangle
$$

we see that the problems appear when the boundaries certainly exist: $\partial \Sigma \neq 0$ no momentum map. Consequently the problem can be solved from the point of view of the Atyah-Bott theorem redefining the symplectic structure with the help of the moduli-space of the flat connections. (in a future work [51] this problem will be explicitly exemplified).

\section{Invariant $S O(2,4)$ Action and Breakdown Mechanism}

The explicit construction given recently [73] of geometrical lagrangians based in a group manifold with conformal structure is reviewed here in order to understand how it can be connected with the general dynamics and quantization procedures.

\section{Linear in $R^{A B}$}

$$
S=\int \mu_{A B} \wedge R^{A B}
$$

in this case we note first, that the $S O(2,4)$-valuated tensor $\mu_{A B}$ acts as multiplier in $S$ (without any role in dynamics, generally speaking). Having this fact in mind, let us consider the following points.

(i) If we have two diffeomorphic (or gauge) nonequivalent $S O(2,4)$-valuated connections, namely $\Gamma^{A B}$ and $\widetilde{\Gamma}^{A B}$, their difference transforms as a second rank six-tensor under the action of $S O(2,4)$

$$
\begin{aligned}
\kappa^{A B} & =G_{C}^{A} G_{D}^{B}{ }^{\kappa^{C D}}, \\
\kappa^{A B} & \equiv \widetilde{\Gamma}^{A B}-\Gamma^{A B} .
\end{aligned}
$$

(ii) If we now calculate the curvature from $\widetilde{\Gamma}^{A B}$ we obtain

$$
\widetilde{R}^{A B}=R^{A B}+\mathcal{D} \kappa^{A B},
$$

where the $S O(2,4)$ covariant derivative is defined in the usual way

$$
\mathcal{D} \kappa^{A B}=d \kappa^{A B}+\Gamma_{C}^{A} \wedge \kappa^{C B}+\Gamma_{D}^{B} \wedge \kappa^{A D} .
$$


(iii) Redefining the $S O(2,4)$ six vectors as $V_{2}^{A} \equiv \psi^{A}$ and $V_{1}^{B} \equiv \varphi^{B}$ (in order to put all in the standard notation), the 2-form $\kappa^{A B}$ can be constructed as

$$
\kappa^{A B} \rightarrow \psi^{[A} \varphi^{B]} d U .
$$

Then we introduce all into the $\widetilde{R}^{A B}$ ( $U$ scalar function) and get

$$
\begin{aligned}
\widetilde{R}^{A B} & =R^{A B}+\mathcal{D}\left(\psi^{[A} \varphi^{B]} d U\right) \\
& =R^{A B}+\left(\psi^{[A} \mathcal{D} \varphi^{B]}-\varphi^{[A} \mathcal{D} \psi^{B]}\right) \wedge d U .
\end{aligned}
$$

The next step is to find the specific form of $\mu_{A B}$ such that $\tilde{\mu}_{A B}=\mu_{A B}$ (invariant under tilde transformation) in order to make the splitting of the transformed action $\widetilde{S}$ weakly reductive as follows.

(iv) Let us define

$$
\widetilde{\theta}^{A}=\widetilde{\mathcal{D}} \varphi^{A}
$$

with the connection $\widetilde{\Gamma}^{A B}=\Gamma^{A B}+\kappa^{A B}$, then

$$
\begin{aligned}
& \tilde{\theta}^{A}=\underbrace{\mathcal{D} \varphi^{A}}_{\theta^{A}}+\kappa_{B}^{A} \varphi^{B}, \\
& \tilde{\theta}^{A}=\theta^{A}+\left[\psi^{A}\left(\varphi^{B}\right)^{2}-\varphi^{A}(\psi \cdot \varphi)\right] \wedge d U,
\end{aligned}
$$

where $\left(\varphi^{B}\right)^{2}=\left(\varphi_{B} \varphi^{B}\right)$ and $(\psi \cdot \varphi)=\psi_{B} \varphi^{B}$ etc.

In the same manner we also define

$$
\begin{aligned}
& \tilde{\eta}^{A}=\widetilde{\mathcal{D}} \psi^{A}, \\
& \tilde{\eta}^{A}=\eta^{A}+\left[\psi_{2}^{A}(\psi \cdot \varphi)-\varphi^{A}\left(\psi^{B}\right)^{2}\right] \wedge d U .
\end{aligned}
$$

(v) To determine $\mu_{A B}$ we propose to cast it in the form

$$
\mu_{A B} \propto \rho_{s}\left[a \psi^{F} \varphi^{E} \epsilon_{A B C D E F}\left(\theta^{C} \wedge \eta^{D}+\theta^{C} \wedge \theta^{D}+\eta^{C} \wedge \eta^{D}\right)+b \kappa^{A B}\right]
$$

with $\rho_{S}, a, b$ scalar functions in particular contractions of vectors and bivectors $S O(2,4)$-valuated with $\left.\epsilon_{A B C D E F}\right)$ to be determined. The behaviour under the tilde transformation is

$$
\tilde{\mu}_{A B} \propto \mu_{A B}-\frac{1}{2} \rho_{s} a \psi^{F} \varphi^{E} \epsilon_{A B E F} d \xi \wedge d U,
$$

where $\xi=\left(\psi^{A}\right)^{2}\left(\varphi^{B}\right)^{2}-(\psi \cdot \varphi)^{2}$.

(vi) Finally we have to look at the behaviour of the transformed action

$$
\begin{aligned}
\widetilde{S} & =\int \widetilde{\mu}_{A B} \wedge \widetilde{R}^{A B} \\
& =S+\int \frac{1}{2} \rho_{S} a \kappa_{A B} \wedge R^{A B} \wedge d \xi+\int \mu_{A B} \wedge \mathcal{D} \kappa^{A B} .
\end{aligned}
$$

We see that till this point, the $S O(2,4)$-valuated six-vectors $\psi^{F}$ and $\varphi^{E}$ are in principle arbitrary. However, under the conditions discussed in the first Section the vectors go to the fiducial ones modulo a phase. Consequently

$$
\xi \rightarrow A^{2} B^{2}
$$


and the bivector comes to

$$
\kappa^{A B} \rightarrow \psi^{[A} \varphi^{B]} d U \rightarrow \Delta(A B) \epsilon^{\alpha \beta}=\alpha \beta A B \epsilon^{\alpha \beta}=A B \epsilon^{\alpha \beta}, \quad \alpha, \beta: 5,6,
$$

where we define the 2nd rank antisymmetric tensor $\epsilon^{\alpha \beta}$ and

$$
\Delta=\operatorname{Det}\left(\begin{array}{cc}
\lambda^{*} \alpha & -\mu \beta \\
-\mu^{*} \alpha & \lambda \beta
\end{array}\right)=\alpha \beta=1 \text { (unitary transformation) }
$$

Below we consider two important cases with respect to the components $m$ and $\lambda$.

\section{$A=m$ and $B=\lambda$}

1. If the coefficients $A=m$ and $B=\lambda$ play the role of constant parameters we have

$$
d \xi \rightarrow d\left(\lambda^{2} m^{2}\right)=0
$$

and

$$
\mathcal{D} \kappa^{A B} \rightarrow d(\lambda m) \epsilon^{\alpha \beta} \wedge d U=0
$$

making the original action $S$ invariant, e.g.

$$
\left.\widetilde{S}\right|_{V_{0}}=\int \tilde{\mu}_{A B} \wedge \widetilde{R}^{A B}=\int \mu_{A B} \wedge R^{A B}=S
$$

being $\left.\widetilde{S}\right|_{V_{0}}$ the restriction of $\widetilde{S}$ under the subspace generated by $V_{0}$ and consequently breaking the symmetry from $S O(2,4) \rightarrow S O(1,3)$.

2 . The connections after the symmetry breaking (when the mentioned conditions with $\lambda$ and $m$ constants are fulfilled) become

$$
\begin{gathered}
\widetilde{\Gamma}^{A B}=\Gamma^{A B}+\kappa^{A B} \Rightarrow \text { b.o.s. } \rightarrow \widetilde{\Gamma}^{i j}=\Gamma^{i j} ; \widetilde{\Gamma}^{i 5}=\Gamma^{i 5}, \quad \widetilde{\Gamma}^{i 6}=\Gamma^{i 6}, \\
\text { but } \quad \widetilde{\Gamma}^{56}=\Gamma^{56}-(\lambda m) d U .
\end{gathered}
$$

3. Vectors $\widetilde{\theta}^{A}$ and $\widetilde{\eta}^{A}$ after the symmetry breaking and under the same conditions become

and evidently $\mu_{i 5}=\mu_{i 6}=0$.

$$
\begin{aligned}
& \widetilde{\theta}^{A}=\underbrace{d \varphi^{A}+\Gamma_{C}^{A} \wedge \varphi^{C}}_{\theta^{A}}+\kappa_{B}^{A} \varphi^{B} \Rightarrow \text { b.o.s., } \\
& \widetilde{\theta}^{i}=\theta^{i}=0+\Gamma_{5}^{i} m+0 \Rightarrow \theta^{i}=\Gamma_{5}^{i} m, \\
& \widetilde{\theta}^{5}=0=0+0=0, \\
& \widetilde{\eta}^{A}=\underbrace{d \psi^{A}+\Gamma_{C}^{A} \wedge \psi^{C}}_{\theta^{A}}+\kappa_{B}^{A} \psi^{B} \Rightarrow \text { b.o.s., } \\
& \widetilde{\eta}^{i}=\eta^{i}=0-\Gamma_{6}^{i} \lambda+0 \Rightarrow \eta^{i}=-\Gamma_{6}^{i} \lambda, \\
& \widetilde{\eta}^{6}=\eta^{6}=0
\end{aligned}
$$


4. Consequently from the last points, curvatures become

$$
\begin{aligned}
& R^{i j}=R_{\{\}}^{i j}+m^{-2} \theta^{i} \wedge \theta^{j}+\lambda^{-2} \eta^{i} \wedge \eta^{j}, \\
& R^{i 5}=m^{-1}[\overbrace{d \theta^{i}+\omega^{i}{ }_{j} \wedge \theta^{j}}^{D \theta^{i}}+\left(\frac{m}{\lambda}\right) \eta^{i} \wedge \Gamma^{65}]=m^{-1}\left[D \theta^{i}-\frac{m}{\lambda} \eta^{i} \wedge \Gamma^{65}\right], \\
& R^{i 6}=-\lambda^{-1}\left[D \eta^{i}-\left(\frac{m}{\lambda}\right)^{-1} \theta^{i} \wedge \Gamma^{56}\right], \\
& R^{56}=d \Gamma^{56}+(m \lambda)^{-1} \theta_{i} \wedge \eta^{i},
\end{aligned}
$$

where $D$ is the $S O(1,3)$ covariant derivative.

5. The tensor responsible for the symmetry breaking becomes

$$
\begin{aligned}
& \mu_{i j}=-2 \rho_{s} a \lambda m \epsilon_{i j k l}\left(\theta^{k} \wedge \eta^{l}+\theta^{k} \wedge \theta^{l}+\eta^{k} \wedge \eta^{l}\right) \\
& \mu_{56}=-\rho_{s} b \epsilon_{56} \lambda m d U .
\end{aligned}
$$

6. Consequently, with all ingredients at hand, the action will be

$$
S=\int \mu_{A B} \wedge R^{A B}=\underbrace{\int \mu_{i j} \wedge R^{i j}}_{S_{1}}+\underbrace{\int \mu_{56} \wedge R^{56}}_{S_{2}},
$$

where

$$
\begin{aligned}
S_{1}= & -2 \int \rho_{s} a \epsilon_{i j k l}\left(\theta^{k} \wedge \eta^{l}+\theta^{k} \wedge \theta^{l}+\eta^{k} \wedge \eta^{l}\right) \wedge\left(\lambda m R_{\{\}}^{i j}+\frac{\lambda}{m} \theta^{i} \wedge \theta^{j}+\frac{m}{\lambda} \eta^{i} \wedge \eta^{j}\right) \\
= & -2 \int \rho_{s} a \epsilon_{i j k l}\left(\theta^{k} \wedge \eta^{l} \wedge \lambda m R_{\{\}}^{i j}+\theta^{k} \wedge \theta^{l} \wedge \lambda m R_{\{\}}^{i j}+\eta^{k} \wedge \eta^{l} \wedge \lambda m R_{\{\}}^{i j}\right) \\
& -2 \int \rho_{s} a \epsilon_{i j k l}\left(\theta^{k} \wedge \eta^{l} \wedge \frac{\lambda}{m} \theta^{i} \wedge \theta^{j}+\theta^{k} \wedge \theta^{l} \wedge \frac{\lambda}{m} \theta^{i} \wedge \theta^{j}+\eta^{k} \wedge \eta^{l} \wedge \frac{\lambda}{m} \theta^{i} \wedge \theta^{j}\right) \\
& -2 \int \rho_{s} a \epsilon_{i j k l}\left(\theta^{k} \wedge \eta^{l} \wedge \frac{m}{\lambda} \eta^{i} \wedge \eta^{j}+\theta^{k} \wedge \theta^{l} \wedge \frac{m}{\lambda} \eta^{i} \wedge \eta^{j}+\eta^{k} \wedge \eta^{l} \wedge \frac{m}{\lambda} \eta^{i} \wedge \eta^{j}\right)
\end{aligned}
$$

and

$$
S_{2}=-\lambda m \int \rho_{s} b \epsilon_{56} \wedge\left(d \Gamma^{56}+(m \lambda)^{-1} \theta_{i} \wedge \eta^{i}\right) .
$$

7. At this point (the mathematical justification will come later) we can naturally associate the tetrad field with the $\theta$-form

$$
\theta^{k} \sim e_{a}^{k} \omega^{a}
$$

consequently a metric can be induced in $M_{4}$ :

$$
\eta_{a b}=g_{j k} e_{a}^{j} e_{b}^{k}, \quad g_{j k}=\eta_{a b} e_{j}^{a} e_{k}^{b}, \quad e_{a}^{k} e_{k}^{b}=\delta_{b}^{a}, \quad \text { etc. },
$$

where $\eta_{j k}$ is the Minkowski metric. That allows us to lift up and to lower down indices, and $\eta^{i}$ with the following symmetry typical of a $S U(2,2)$ Clifford structure

$$
\begin{aligned}
& \eta^{k} \sim f_{a}^{k} \omega^{a}, \\
& e_{j}^{a} f_{a}^{k} g_{l k}=f_{l j}=-f_{j l}
\end{aligned}
$$


that consequently allows us to introduce into the model an electromagnetic field (that will be proportional to $f_{l j}$ ).

8. So we can re-write the action as

$$
\begin{aligned}
S_{1}= & -2 \int \rho_{s} a \epsilon_{i j k l}\left(\theta^{k} \wedge \eta^{l}+\theta^{k} \wedge \theta^{l}+\eta^{k} \wedge \eta^{l}\right) \wedge\left(\lambda m R_{\{\}}^{i j}\right. \\
& \left.+\frac{\lambda}{m} \theta^{i} \wedge \theta^{j}+\frac{m}{\lambda} \eta^{i} \wedge \eta^{j}\right) \\
= & -2 \int \rho_{s} a\left[\lambda m\left(f_{i j} R_{\{\}}^{i j}+\left(g_{i j}+f_{i}^{k} f_{k j}\right) R_{\{\}}^{i j}\right)+\left(\frac{\lambda}{m}+\frac{m}{\lambda}\right) f^{k j} f_{k j}\right. \\
& \left.+\left(\frac{\lambda}{m} \sqrt{g}+\frac{m}{\lambda} \sqrt{f}\right)\right] d^{4} x
\end{aligned}
$$

In the above expression we have taken into account the following:

(i) Terms $\sim \eta \wedge \eta \wedge \eta \wedge \theta$ and $\eta \wedge \theta \wedge \theta \wedge \theta$ vanish;

(ii) Terms $\sim \eta \wedge \eta \wedge \theta \wedge \theta$ and $\eta \wedge \eta \wedge \theta \wedge \theta$ lead to $\rightarrow f^{k j} f_{k j}$;

(iii) Term $\sim \epsilon_{i j k l} \theta^{k} \wedge \eta^{l} \wedge R_{\{\}}^{i j}$ leads $\rightarrow f_{i j} R_{\{\}}^{i j}$ picking the antisymmetric part of the generalized Ricci tensor (containing torsion);

(iv) Term $\sim \epsilon_{i j k l}\left(\theta^{k} \wedge \theta^{l}+\eta^{k} \wedge \eta^{l}\right) R_{\{\}}^{i j}$ leads to $\rightarrow\left(g_{i j}+f_{i}^{k} f_{k j}\right) R_{\{\}}^{i j}$ picking the symmetric part of the generalized Ricci tensor (containing Einstein-Hilbert plus quadratic torsion term);

(v) Terms $\sim \eta \wedge \eta \wedge \eta \wedge \eta$ and $\theta \wedge \theta \wedge \theta \wedge \theta$ lead to the volume elements $\sqrt{f}$ and $\sqrt{g}$, respectively, where we defined as usual $g \equiv \operatorname{Det}\left(g_{l k}\right)$ and $f \equiv \operatorname{Det}\left(f_{l k}\right)=\left(f_{l k}^{*} f^{l k}\right)^{2}$.

\section{$A=m(x)$ and $B=\lambda(x):$ Spontaneous Subspace}

If the coefficients $A=m(x)$ and $B=\lambda(x)$ are not constant but functions of coordinates we have

$$
d \xi \rightarrow d\left(\lambda^{2} m^{2}\right)=2 d(\lambda m)
$$

and

$$
\mathcal{D} \kappa^{A B} \rightarrow d(\lambda m) \epsilon^{\alpha \beta} \wedge d U
$$

Consequently from the following explicit computations

$$
\begin{aligned}
\widetilde{S} & =\int \widetilde{\mu}_{A B} \wedge \widetilde{R}^{A B} \\
& =S+\int \frac{1}{2} \rho_{S} a \kappa_{A B} \wedge R^{A B} \wedge d \xi+\int \mu_{A B} \wedge \mathcal{D} \kappa^{A B} \\
& =S-\int \frac{1}{2} \rho_{s} a R^{A B} \wedge \kappa_{A B} \wedge d \xi+\int \mu_{A B} \wedge \mathcal{D} \kappa^{A B} \\
& =S-\int \frac{1}{2} \rho_{S} a R_{\alpha \beta} \epsilon^{\alpha \beta} \lambda m d U \wedge 2 d(\lambda m)+\int \mu_{\alpha \beta} \epsilon^{\alpha \beta} d(\lambda m) \wedge d U \\
& =S+\int \frac{1}{2} \rho_{S} a R_{\alpha \beta} \epsilon^{\alpha \beta} \lambda m 2 d(\lambda m) \wedge d U+\int \mu_{\alpha \beta} \epsilon^{\alpha \beta} d(\lambda m) \wedge d U, \\
\widetilde{S} & =S+\int\left[\mu_{\alpha \beta}+\rho_{S} a R_{\alpha \beta} \lambda m\right] \epsilon^{\alpha \beta} d(\lambda m) \wedge d U .
\end{aligned}
$$


we obtain the required condition:

$$
\begin{aligned}
\widetilde{S} & =S \quad \text { if } \\
\mu_{\alpha \beta} & =-\rho_{s} a R_{\alpha \beta} \lambda m,
\end{aligned}
$$

then we see that $\mu_{A B}$ takes the place of an induced metric and it is proportional to the curvature

$$
\begin{aligned}
& R_{\alpha \beta}=\Lambda \mu_{\alpha \beta} \\
& \text { with } \quad \Lambda=-\left(\rho_{s} a \lambda m\right)^{-1} .
\end{aligned}
$$

Note that we have now a four-dimensional space-time plus the above "internal" space of a constant curvature. This point is very important as a new compactification-like mechanism.

Remark 1 A geometrical structure defined on the coset $K=G / H$, with $H$ stability group, is defined weakly reductive if there is a vector space $\mathcal{K}$ satisfying the following conditions: $\mathcal{G}=\mathcal{H}+\mathcal{K}$ and $[\mathcal{H}, \mathcal{K}] \subset \mathcal{K}$ being $\mathcal{G}$ and $\mathcal{H}$ the Lie algebras of $G$ and $H$ respectively.

\section{Supergravity as a Gauge Theory and Topological QFT}

In previous works $[57,58]$ we have shown, by means of a toy model, that there exists a supersymmetric analog of the above symmetry breaking mechanism coming from the topological QFT. Here we recall some of the above ideas in order to see clearly the analogy between the group structures of the simplest supersymmetric case, Osp (4), and of the classical conformal group $S O(2,4)$.

The starting point is the super $S L(2 C)$ superalgebra (strictly speaking $O s p(4)$ )

$$
\begin{aligned}
& {\left[M_{A B}, M_{C D}\right]=\epsilon_{C}\left({ }_{A} M_{B}\right)_{D}+\epsilon_{D}\left({ }_{A} M_{B}\right)_{C},} \\
& {\left[M_{A B}, Q_{C}\right]=\epsilon_{C}\left({ }_{A} Q_{B}\right), \quad\left\{Q_{A}, Q_{B}\right\}=2 M_{A B} .}
\end{aligned}
$$

Here the indices $A, B, C, \ldots$ stay for $\alpha, \beta, \gamma \ldots(\dot{\alpha}, \dot{\beta}, \dot{\gamma} \ldots)$ spinor indices: $\alpha, \beta(\dot{\alpha}, \dot{\beta})=$ $1,2(\dot{1}, \dot{2})$ in the Van der Werden spinor notation. We define the superconnection $A$ due the following "gauging"

$$
A^{p} T_{p} \equiv \omega^{\alpha \dot{\beta}} M_{\alpha \dot{\beta}}+\omega^{\alpha \beta} M_{\alpha \beta}+\omega^{\dot{\alpha} \dot{\beta}} M_{\dot{\alpha} \dot{\beta}}+\omega^{\alpha} Q_{\alpha}-\omega^{\dot{\alpha}} \bar{Q}_{\dot{\alpha}},
$$

where $(\omega M)$ defines a ten-dimensional bosonic manifold ${ }^{1}$ and $p \equiv$ multi-index, as usual. Analogically the super-curvature is defined by $F \equiv F^{p} T_{p}$ with the following detailed structure

$$
\begin{aligned}
F(M)^{A B} & =d \omega^{A B}+\omega_{C}^{A} \wedge \omega^{C B}+\omega^{A} \wedge \omega^{B}, \\
F(Q)^{A} & =d \omega^{A}+\omega_{C}^{A} \wedge \omega^{C} .
\end{aligned}
$$

From (46) it is easy to see that there are a bosonic part and a fermionic one associated with the even and odd generators of the superalgebra. Our proposal for the "toy" action was (as before for $S O(2,4))$ as follows:

$$
S=\int F^{p} \wedge \mu_{p}
$$

\footnotetext{
${ }^{1}$ Corresponding to the number of generators of $S O(4,1)$ or $S O(3,2)$ that define the group manifold
} 
where the tensor $\mu_{p}$ (that plays the role of a $O s p(4)$ diagonal metric as in the Mansouri proposal) is defined as

$$
\mu_{\alpha \dot{\beta}}=\zeta_{\alpha} \wedge \bar{\zeta}_{\dot{\beta}} \mu_{\alpha \beta}=\zeta_{\alpha} \wedge \zeta_{\beta} \mu_{\alpha}=v \zeta_{\alpha} \text { etc }
$$

with $\zeta_{\alpha}\left(\bar{\zeta}_{\dot{\beta}}\right)$ anti-commuting spinors (suitable basis) ${ }^{2}$ and $v$ the parameter of the breaking of super $S L(2 C)(O s p(4))$ to $S L(2 C)$ symmetry of $\mu_{p}$. Note that the introduction of the parameter $v$ means that we do not take care of the particular dynamics to break the symmetry.

In order to obtain dynamical equations of the theory, we proceed to perform variation of the proposed action (49)

$$
\begin{aligned}
\delta S & =\int \delta F^{p} \wedge \mu_{p}+F^{p} \wedge \delta \mu_{p} \\
& =\int d_{A} \mu_{p} \wedge \delta A^{p}+F^{p} \wedge \delta \mu_{p},
\end{aligned}
$$

where $d_{A}$ is the exterior derivative with respect to the super-SL (2C) connection and $\delta F=$ $d_{A} \delta A$ have been used. Then, as the result, the dynamics is described by

$$
d_{A} \mu=0, \quad F=0 .
$$

The fist equation claims that $\mu$ is covariantly constant with respect to the super $S L(2 C)$ connection. This fact will be very important when the super $S L(2 C)$ symmetry breaks down to $S L$ (2C) because $d_{A} \mu=d_{A} \mu_{A B}+d_{A} \mu_{A}=0$, a soldering form will appear. The second equation gives the condition for a super Cartan connection $A=\omega^{A B}+\omega^{A}$ to be flat, as it is easy to see from the reductive components of above expressions

$$
\begin{aligned}
F(M)^{A B} & =R^{A B}+\omega^{A} \wedge \omega^{B}=0, \\
F(Q)^{A} & =d \omega^{A}+\omega_{C}^{A} \wedge \omega^{C}=d_{\omega} \omega^{A}=0,
\end{aligned}
$$

where now $d_{\omega}$ is the exterior derivative with respect to the $S L(2 C)$ connection and $R^{A B} \equiv$ $d \omega^{A B}+\omega_{C}^{A} \wedge \omega^{C B}$ is the $S L(2 C)$ curvature. Then

$$
F=0 \Leftrightarrow R^{A B}+\omega^{A} \wedge \omega^{B}=0 \quad \text { and } \quad d_{\omega} \omega^{A}=0
$$

the second condition says that the $S L(2 C)$ connection is super-torsion free. The first doesn't say that the $S L(2 C)$ connection is flat, but it claims that it is homogeneous with a cosmological constant related to the explicit structure of the Cartan forms $\omega^{A}$, as we will see when the super $S L(2 C)$ action is reduced to the Volkov-Pashnev model [44,45].

\section{Quadratic in $R^{A B}$}

The previous action, linear in the generalized curvature, has some drawbacks that make necessary introduction of additional "subsidiary conditions" due to the fact that the curvatures $R^{i 5}$ and $R^{i 6}$ don't play any role in the linear/first order action. Such curvatures have a very important information about the dynamics of $\theta$ and $\eta$ fields. In order to simplify the equations

${ }^{2}$ In general this tensor has the same structure as the Cartan-Killing metric of the group under consideration. 
of motion we define

$$
\begin{aligned}
\Gamma^{56} & \equiv A, \\
m^{-1} \theta^{i} & \equiv \widetilde{\theta}^{i}, \\
\lambda^{-1} \eta^{i} & \equiv \tilde{\eta}^{i},
\end{aligned}
$$

and as always

$$
R^{i j}=R_{\{\}}^{i j}+m^{-2} \theta^{i} \wedge \theta^{j}+\lambda^{-2} \eta^{i} \wedge \eta^{j}
$$

with the $S O(1,3)$ curvature $R_{\{\}}^{i j}=d \omega^{i j}+\omega_{\lambda}^{i} \wedge \omega^{\lambda j}$. Consequently from the quadratic Lagrangian density

$$
S=\int R_{A B} \wedge R^{A B}
$$

we obtain the following equations of motion:

$$
\begin{aligned}
& \frac{\delta\left(R_{A B} \wedge R^{A B}\right)}{\delta \theta^{i}} \rightarrow D\left(D \tilde{\theta}_{j}\right)+2 R_{i j} \wedge \widetilde{\theta}^{i}-\tilde{\theta}^{i} \wedge \tilde{\eta}_{i} \wedge \tilde{\eta}_{j}+\tilde{\theta}_{j} \wedge A \wedge A=0, \\
& \frac{\delta\left(R_{A B} \wedge R^{A B}\right)}{\delta \eta^{i}} \rightarrow D\left(D \widetilde{\eta}_{j}\right)+2 R_{j k} \wedge \widetilde{\eta}^{k}-\widetilde{\theta}^{i} \wedge \widetilde{\eta}_{i} \wedge \widetilde{\theta}_{j}+\widetilde{\eta}_{j} \wedge A \wedge A=0, \\
& \frac{\delta\left(R_{A B} \wedge R^{A B}\right)}{\delta \Gamma^{56}} \rightarrow \widetilde{\theta}^{i} \wedge \widetilde{\theta}_{i}=\widetilde{\eta}^{i} \wedge \tilde{\eta}_{i}, \\
& \frac{\delta\left(R_{A B} \wedge R^{A B}\right)}{\delta \omega_{j}^{i}} \rightarrow-D R_{k l}+D \widetilde{\theta}_{k} \wedge \widetilde{\theta}_{l}+D \widetilde{\eta}_{k} \wedge \tilde{\eta}_{l}+\widetilde{\theta}_{k} \wedge \tilde{\eta}_{l} \wedge A=0 .
\end{aligned}
$$

\section{Maxwell Equations and the Electromagnetic Field}

As we claimed before we can identify

$$
\begin{aligned}
\theta^{i} & \equiv e_{\mu}^{i} d x^{\mu}, \\
\eta^{i} & \equiv f_{\mu}^{i} d x^{\mu}
\end{aligned}
$$

with the symmetries

$$
e_{\mu}^{i} e_{i}^{\nu}=\delta_{\mu}^{\nu}, e_{\mu}^{i} e_{i v}=g_{\mu \nu}=g_{\nu \mu}
$$

and

$$
f_{\mu}^{i} f_{i}^{\nu}=\delta_{\mu}^{\nu}, \quad e_{i v} f_{\mu}^{i}=f_{\mu \nu}=-f_{\nu \mu}
$$

such that the geometrical (Bianchi) condition

$$
\nabla_{[\rho} f_{\mu \nu]}=\nabla_{\rho}^{*} f^{\rho v}=0
$$

or in the language of differential forms

$$
D\left(\widetilde{\theta}^{i} \wedge \widetilde{\eta}_{i}\right)=0
$$

holds, thus the curvatures $R^{i 6}$ and $R^{i 5}$ are enforced to be null. And conversely if $R^{i 6}$ and $R^{i 5}$ are zero then $D\left(\widetilde{\theta}^{i} \wedge \widetilde{\eta}_{i}\right)=0$ or equivalently $\nabla_{[\rho} f_{\mu \nu]}=\nabla_{\rho}^{*} f^{\rho v}=0$.

\section{Springer}


Proof From expressions $(28,29)$, namely: $R^{i 5}=\left[D \widetilde{\theta}^{i}-\widetilde{\eta}^{i} \wedge \Gamma^{65}\right]$ and $R^{i 6}=\left[-D \widetilde{\eta}^{i}+\right.$ $\left.\tilde{\theta}^{i} \wedge \Gamma^{56}\right]$ we make

$$
\begin{aligned}
& R^{i 5} \wedge \tilde{\eta}_{i}+\tilde{\theta}_{i} \wedge R^{i 6}=D\left(\widetilde{\theta}^{i} \wedge \tilde{\eta}_{i}\right)+\left(\widetilde{\eta}^{i} \wedge \Gamma^{56}\right) \wedge \tilde{\eta}_{i}+\widetilde{\theta}_{i} \wedge\left(\widetilde{\theta}^{i} \wedge \Gamma^{56}\right), \\
& R^{i 5} \wedge \tilde{\eta}_{i}+\tilde{\theta}_{i} \wedge R^{i 6}=D\left(\widetilde{\theta}^{i} \wedge \tilde{\eta}_{i}\right) .
\end{aligned}
$$

In the last line we used the constraint given by Eq. (62) Consequently if $R^{i 6}$ and $R^{i 5}$ are zero, then $D\left(\widetilde{\theta}^{i} \wedge \tilde{\eta}_{i}\right)=0$ or equivalently $\nabla_{[\rho} f_{\mu \nu]}=\nabla_{\rho}^{*} f^{\rho v}=0$ and vice versa.

Corollary 2 Note that the vanishing of the $R^{56}$ curvature (that transforms as a Lorentz scalar) does not modify the equation of motion for $\Gamma^{56}$ and simultaneously defines the electromagnetic field as

$$
\begin{aligned}
& R^{56}=d \Gamma^{56}+(m \lambda)^{-1} \theta_{i} \wedge \eta^{i}=0, \\
& \Rightarrow d A-F=0 .
\end{aligned}
$$

\section{Equations of Motion in Components and Symmetries}

Let us define

$$
\begin{aligned}
& R_{\{\} \mu \nu}^{i j}=\partial_{\mu} \omega_{\nu}^{i j}-\partial_{\nu} \omega_{\mu}^{i j}+\omega_{\mu k}^{i} \omega_{\nu}^{k j}-\omega_{\mu}^{k j} \omega_{\nu k}^{i}, \\
& T_{\mu \nu}^{i}=\partial_{\mu} e_{\nu}^{i}-\partial_{\nu} e_{\mu}^{i}+\omega_{\mu k}^{i} e_{\nu}^{k}-\omega_{\nu k}^{i} e_{\mu}^{k}, \\
& S_{\mu \nu}^{i}=\partial_{\mu} f_{\nu}^{i}-\partial_{\nu} f_{\mu}^{i}+\omega_{\mu k}^{i} f_{\nu}^{k}-\omega_{\nu k}^{i} f_{\mu}^{k} .
\end{aligned}
$$

Note that $S_{\mu \nu}^{i}$ is a totally antisymmetric torsion field due the symmetry of $f_{v}^{i} d x^{\nu} \equiv \eta^{i}$. Consequently the equations of motion in components become

$$
\begin{aligned}
& \nabla_{\mu}\left[\sqrt{|g|} R^{i j \mu \nu}\right]+\sqrt{|g|}\left(-m^{-2} T^{j i v}+\lambda^{-2} S^{j i v}\right)-\sqrt{|g|}(\lambda m)^{-1} f^{[i v} A^{i]}=0, \\
& \nabla_{\mu}\left[\sqrt{|g|}\left(R_{\{\}}^{i j \mu \nu}-m^{-2} e^{[i \mu} e^{j] v}+\lambda^{-2} f^{[i \mu} f^{j] v}\right)\right] \\
& \quad+\sqrt{|g|}\left(-m^{-2} T^{j i v}+\lambda^{-2} S^{j i v}\right)-\sqrt{|g|}(\lambda m)^{-1} f^{[i v} A^{i]}=0, \\
& \nabla_{\mu}\left(\sqrt{|g|} T^{j \mu v}\right)+\sqrt{|g|}\left(R_{0\}}^{j v}-m^{-2} e^{j v}+A^{i} A^{v}\right)=0, \\
& \nabla_{\mu}\left(\sqrt{|g|} S^{j \mu i}\right)+\sqrt{|g|}\left(R_{\{\}}^{i j}-\lambda^{-2} f^{i j}+A^{[i} A^{j]}\right)=0, \\
& \nabla_{[\mu} A_{\nu]}=F_{\mu \nu}=(\lambda m)^{-1} F_{\mu \nu}, \\
& \nabla_{[\rho} F_{\mu \nu]}=0 .
\end{aligned}
$$

\section{Nonlinear Realizations Viewpoint}

Note that in our case Eqs. $(64,65)$ identify $\theta^{i} \sim e^{i}$ and $\eta^{i} \sim f^{i}$ making the table below completely clear. Note that $\Gamma^{65}$ is identified with the $\mathbf{g}$ of Ivanov and Niederle $[14,15]$.

Algebra and transformations in the case of the work of Ivanov and Niederle are different due different definitions of the generators of the $S O(2,4)$ algebra, however the meaning of $\mathrm{g}$ which is associated to the connection $\Gamma^{65}$ remains obscure for us because of the second 
This work

$[14,15]$

\begin{tabular}{lll}
\hline$R^{i j}$ & $R_{\{\}}^{i j}+m^{-2} \theta^{i} \wedge \theta^{j}+\lambda^{-2} \eta^{i} \wedge \eta^{j}$ & $R_{\{\}}^{i j}+4 g e^{i} \wedge f^{j}$ \\
$R^{i 5}$ & $m^{-1}\left[D \theta^{i}-\frac{m}{\lambda} \eta^{i} \wedge \Gamma^{65}\right]$ & $D e^{i}+2 g e^{i} \wedge \mathbf{g}$ \\
$R^{i 6}$ & $-\lambda^{-1}\left[D \eta^{i}-\left(\frac{m}{\lambda}\right)^{-1} \theta^{i} \wedge \Gamma^{56}\right]$ & $D f^{i}-2 g f^{i} \wedge \mathbf{g}$ \\
$R^{56}$ & $d \Gamma^{56}+(m \lambda)^{-1} \theta_{i} \wedge \eta^{i}$ & $d \mathbf{g}+4 g e_{i} \wedge f^{i}$ \\
DS/ADS reduction & Yes & No \\
\hline
\end{tabular}

Cartan structure equations $R^{i 5}$ and $R^{i 6}$. Note that, although the group theoretical viewpoint in the case of the simultaneous nonlinear realization of the affine and conformal group [55,56] to obtain Einstein gravity are more or less clear, the pure geometrical picture is still hard to recognize due the factorization problem and the orthogonality between coset elements and the corresponding elements of the stability subgroup.

\section{Symplectic Structures, Poisson Manifolds and Noncommutativity}

\section{Generalization of Rothstein's Theorems Even Supersymplectic Supermanifols}

The existence of a (super) symplectic structure on a manifold is a very significant constraint and many simple and natural constructions in symplectic geometry lead to manifolds which cannot possess a symplectic structure (or to spaces which cannot possess a manifold structure). However these spaces often inherit a bracket of functions from the Poisson bracket on the original symplectic manifold. It is a (semi-)classical limit of quantum theory and also is the theory dual to Lie algebra theory and, more generally, to Lie algebroid theory.

Poisson structures are the first stage in quantization, in the specific sense that a Poisson bracket is the first term in the power series of a deformation quantization. Poisson groups are also important in studies of complete integrability.

From the point of view of the Poisson structure associated to the differential forms induced by the unitary transformation from the G-valuated tangent space implies automatically, the existence of an even non-degenerate (super)metric. The remaining question of the previous section was if the induced structure from the tangent space (via Ambrose-Singer theorem) was intrinsically related to a supermanifold structure (e.g. noncommutativity, hidden supersymmetry, etc.). Some of these results were pointed out in the context of supergeometrical analysis by Rothstein and by others authors [61-63], corroborating this fact in some sense. Consequently we have actually several models coming mainly from string theoretical frameworks that are potentially ruled out $[66,70]$. Let us review and develop our earlier work [59] to work out this issue with more detail: from the structure of the tangent space $T_{p}(M)$ we have seen

$$
\begin{aligned}
U_{A}^{B}(P) & =\delta_{A}^{B}+\mathcal{R}_{A \mu \nu}^{B} d x^{\mu} \wedge d x^{\nu} \\
& =\delta_{A}^{B}+\omega^{k}\left(\mathcal{T}_{k}\right)_{A}^{B}
\end{aligned}
$$

where the Poisson structure is evident (as the dual of the Lie algebra of the group manifold) in our case leading to the identification

$$
\mathcal{R}_{A \mu \nu}^{B} d x^{\mu} \wedge d x^{\nu} \equiv \omega^{k}\left(\mathcal{T}_{k}\right)_{A}^{B}
$$


We have in the general case, a (matrix) automorphic structure. The general translation to the spacetime from the above structure in the tangent space takes the form

$$
\begin{aligned}
\widetilde{\omega}= & \frac{1}{2}\left[\omega_{i j}+\frac{1}{2}\left(\omega_{k l}\left(\Gamma_{a i}^{k} \Gamma_{b j}^{l}-\Gamma_{b j}^{k} \Gamma_{a i}^{l}\right)+g_{b d} R_{i j a}^{d}\right) d \psi^{a} d \psi^{b}\right] d x^{i} \wedge d x^{j} \\
& +\omega_{i j} A_{b m}^{j} d x^{m} d x^{i} d \psi^{b}+ \\
& +\frac{1}{2}\left[g_{a b}+\frac{1}{2}\left(g_{c d}\left(\Gamma_{i b}^{c} \Gamma_{j a}^{d}-\Gamma_{j a}^{c} \Gamma_{i b}^{d}\right)+\omega_{l j} R_{a b i}^{l}\right) d x^{i} \wedge d x^{j}\right] d \psi^{a} d \psi^{b} \\
& +g_{a b} A_{i d}^{b} d \psi^{d} d \psi^{a} d x^{i}
\end{aligned}
$$

Because covariant derivatives are defined in the usual (group theoretical) way

$$
\begin{aligned}
D \psi^{a} & =d \psi^{a}-\Gamma_{i b}^{i} d \psi^{b} d x^{i} \\
D x^{i} & =d x^{i}-\Gamma_{a j}^{i} d x^{j} d \psi^{a}
\end{aligned}
$$

we can rewrite $\widetilde{\omega}$ in a compact form as

$$
\begin{aligned}
\tilde{\omega}= & \frac{1}{2}\left[\left(\omega_{i j} D x^{i} \wedge D x^{j}+\frac{1}{2} g_{b d} R_{i j a}^{d} d \psi^{a} d \psi^{b} d x^{i} \wedge d x^{j}\right)\right. \\
& \left.+\left(g_{a b} D \theta^{a} D \theta^{b}+\frac{1}{2} \omega_{l j} R_{a b i}^{l} d x^{i} \wedge d x^{j} d \theta^{a} d \theta^{b}\right)\right]
\end{aligned}
$$

At the tangent space, where that unitary transformation makes the link, the first derivatives of the metric are zero, remaining only the curvatures, we arrive to

$$
\widetilde{\omega}=\frac{1}{2}\left[\left(\eta_{i j}+\frac{1}{2} \epsilon_{b d} R_{i j a}^{d} d \psi^{a} d \psi^{b}\right) d x^{i} \wedge d x^{j}+\left(\epsilon_{a b}+\frac{1}{2} \eta_{l j} R_{a b i}^{l} d x^{i} \wedge d x^{j}\right) d \psi^{a} d \psi^{b}\right]
$$

Here the Poisson structure can be checked

$$
\begin{aligned}
\eta_{i j}+\frac{1}{2} \epsilon_{b d} R_{i j a}^{d} d \psi^{a} d \psi^{b} & =\left(\delta_{j}^{k}+\frac{1}{2} \epsilon_{b d} \eta^{k l} R_{l j a}^{d} d \psi^{a} d \psi^{b}\right) \eta_{k i} \\
\epsilon_{a b}+\frac{1}{2} \eta_{l j} R_{a b i}^{l} d x^{i} \wedge d x^{j} & =\left(\delta_{b}^{c}+\frac{1}{2} \eta_{l j} \epsilon^{c d} R_{d b i}^{l} d x^{i} \wedge d x^{j}\right) \epsilon_{a c}
\end{aligned}
$$

In expressions (80-86) the curvatures, the differential forms and the other geometrical operators depend also on the field where they are defined: $\mathbb{R}, \mathbb{C}$ or $\mathbb{H}$. In the quaternionic $\mathbb{H}$-case the metric is quaternion valuated with the propierty $\omega_{[i j]}^{\dagger}=-\omega_{[j i]}$ and the covariant derivative can be straightforwardly defined as expressions $(81,82)$ but with the connection and coordinates also quaternion valuated. The fundamental point in a such a case going towards a fully reliable gravitational theory is to fix the connection in order to have a true link with the physical situation. The matrix representation of structures $(85,86)$ are automorphic ones: e.g. they belong to the identity and to the symplectic block generating the corresponding trascendent (parameter depending) functions. Now, we will analize the above fundamental structure under the light of the supersymplectic structure given by Rothstein (notation as in Ref. [62,63])

$$
\widetilde{\omega}=\frac{1}{2}\left(\omega_{i j}+\frac{1}{2} g_{b d} R_{i j a}^{d} \theta^{a} \theta^{b}\right) d x^{i} d x^{j}+g_{a b} D \theta^{a} D \theta^{b}
$$


where the usual set of Grassmann supercoordinates were introduced: $x^{1}, \ldots x^{j} ; \theta^{1} \ldots \theta^{d}$; the superspace metrics were defined as: $\omega_{i j}=\left(\frac{\partial}{\partial x^{i}}, \frac{\partial}{\partial x^{j}}\right), g_{a b}=\left(\frac{\partial}{\partial \theta^{a}}, \frac{\partial}{\partial \theta^{b}}\right)$ and

$$
\nabla_{\frac{\partial}{\partial x^{i}}}\left(\theta^{a}\right)=A_{i b}^{i} \theta^{b}
$$

Due to the last expression, we can put $\widetilde{\omega}$ in a compact form with the introduction of a suitable covariant derivative: $D \theta^{a}=d \theta^{a}-A_{i b}^{i} \theta^{b} d x^{i}$. With all the definitions at hands, the Poisson structure of $\widetilde{\omega}$ in the case of Rothstein's is easily verified

$$
\omega_{i j}+\frac{1}{2} g_{b d} R_{i j a}^{d} \theta^{a} \theta^{b}=(\delta_{i}^{k}+\underbrace{\frac{1}{2} g_{b d} \omega^{l k} R_{i l a}^{d} \theta^{a} \theta^{b}}_{\equiv B}) \omega_{k j}
$$

The important remark of Rothstein [62] is that the matrix representation of the structure $B$ has nilpotent entries, schematically

$$
\widetilde{\omega}^{-1}=\left[\omega^{-1}\left(I-B+B^{2}-B^{3} \ldots\right)\right]^{i j} \nabla_{i} \wedge \nabla_{j}+g^{a b} \frac{\partial}{\partial \theta^{a}} \wedge \frac{\partial}{\partial \theta^{b}}
$$

where, as is obvious $B^{n}=0$ for $n>1$ and $n \in \mathbb{N}$.

Remarks from the above analysis, we can compare the Rothstein case with the general one arriving to the following points:

(i) In the Rothstein case only a part of the full induced metric from the tangent space is preserved (“one way" extension [62-65,67-69])

(ii) The geometrical structures (particularly, the fermionic ones) are extended "by hand" motivated, in general, to give by differentiation of the corresponding closed forms, the standard supersymmetric spaces (e.g. Kahler, $C P^{n}$, etc.)[62,63]. In fact it is easily seen from the structure of the covariant derivatives: in the Rothstein case there are Grassmann coordinates instead of the coordinate differential 1-forms contracted with the connection.

(iii) In the Rothstein case the matrix representation (73) coming from the Poisson structure is nilpotent (characteristic of Grassmann manifolds) in sharp contrast with the general representation (68-70) coming from the tangent space of the UFT that is automorphic.

Remark 3 was noted in $[64,65]$ that the following facts arise: (i) A Grassmann algebra, as used in supersymmetry, is equivalent, in some sense, to the spin representation of a Clifford algebra. (ii) The questions about the nature and origin of the vector space on which this orthogonal group acts are completely open. (iii) If it is a tangent space or the space of a local internal symmetry, the vectors will be functions of space-time, and the Clifford algebra will be local. (iv) In other cases we will have a global Clifford algebra. Consequently, the geometric structure of the UFT presented here falls precisely in such a case.

\section{Tangent Space and Even Supermanifold Structure}

The very general QFT structure induced from the tangent space by means of the AmbroseSinger [60] theorem $(78,79)$ verifies straigforwardly the Darboux-Kostant theorem: e.g. it has a supermanifold structure (even in the noncommutative case). Darboux-Kostant's theorem [61] is the supersymmetric generalization of Darboux's theorem and statement that:

Given a $(2 n \mid q)$-dimensional supersymplectic supermanifold $\left(M, \mathcal{A}_{M}, \omega\right)$, it states that for any open neighbourhood $U$ of some point $m$ in $M$ there exists a set $\left(q_{1}, \ldots, q_{n}, p 1, \ldots, p n\right.$; $\left.\xi_{1}, \ldots, \xi_{q}\right)$ of local coordinates on $V \mathcal{E}(U)$ so that $\omega$ on $U$ can be written in the following 
form,

$$
\left.\omega\right|_{U} \equiv \widetilde{\omega}=\sum_{i=1}^{n} d p i \wedge d q^{i}+\sum_{a=1}^{q} \frac{\epsilon}{2}\left(\xi^{a}\right)^{2}, \quad(\epsilon= \pm 1)
$$

Proof by simple inspection we can easily see that the expression (68) has the structure (75). That means that we have locally a supersymplectic vector superspace induced (globally) by a supersymplectic supermanifold.

\section{The Geometrical Reduction and Even Symplectic Super-Metrics}

\section{Example: Volkov-Pashnev Metric}

The super-metric under consideration, proposed by Volkov and Pashnev in [44,45], is the simplest example of symplectic (super) metrics induced by the symmetry breaking from a pure topological first order action. It can be obtained from the $O s p(4)$ (super $S L(2 C)$ ) action via the following procedure.

(i) The Inönu-Wigner contraction [46] in order to pass from $S L(2 C)$ to the super-Poincare algebra (corresponding to the original symmetry of the model of Refs. [44,45,47-49]) then, the even part of the curvature is split into a $\mathbb{R}^{3,1}$ part $R^{\alpha \dot{\beta}}$ and a $S O(3,1)$ part $R^{\alpha \beta}\left(R^{\dot{\alpha} \dot{\beta}}\right)$ associated with the remaining six generators of the original five dimensional $S L(2 C)$ group. This fact is easily realized by knowing that the underlying geometry is reductive: $S L(2 C) \sim S O(4,1) \rightarrow S O(3,1)+\mathbb{R}^{3,1}$. Than we rewrite the superalgebra (45) as

$$
\begin{aligned}
{[M, M] } & \sim M[M, \Pi] \sim \Pi \quad[\Pi, \Pi] \sim M \\
{[M, S] } & \sim S \quad[\Pi, S] \sim S \quad\{S, S\} \sim M+\Pi
\end{aligned}
$$

with $\Pi \sim M_{\alpha \dot{\beta}}, M \sim M_{\alpha \beta}\left(M_{\dot{\alpha} \dot{\beta}}\right)$, and re-scale $m^{2} \Pi=P$ and $m S=Q$. In the limit $m \rightarrow 0$, one recovers the super Poincare algebra. Note that one does not re-scale $M$ since one wants to keep $[M, M] \sim M$ Lorentz algebra, that also is a symmetry of (1).

(ii) The spontaneous breaking of the super $S L(2 C)$ down to the $S L(2 C)$ symmetry of $\mu_{p}$ (e.g. $v \rightarrow 0$ in $\mu_{p}$ ) of such a manner that the even part of the super $S L(2 C)$ action $F(M)^{A B}$ remains.

After these evaluations, it has been explicitly realized that the even part of the original super $S L(2 C)$ action (now a super-Poincare invariant) can be related with the original metric (1) as follows:

$$
R(M)+R(P)+\omega^{\alpha} \omega_{\alpha}-\omega^{\dot{\alpha}} \omega_{\dot{\alpha}} \rightarrow \omega^{\mu} \omega_{\mu}+\mathbf{a} \omega^{\alpha} \omega_{\alpha}-\left.\mathbf{a}^{*} \omega^{\dot{\alpha}} \omega_{\dot{\alpha}}\right|_{V P} .
$$

Note that there is mapping $R(M)+\left.R(P) \rightarrow \omega^{\mu} \omega_{\mu}\right|_{V P}$ that is well defined and can be realized in different forms, and the map of interest here $\omega^{\alpha} \omega_{\alpha}-\omega^{\dot{\alpha}} \omega_{\dot{\alpha}} \rightarrow \mathbf{a} \omega^{\alpha} \omega_{\alpha}-\left.\mathbf{a}^{*} \omega^{\dot{\alpha}} \omega_{\dot{\alpha}}\right|_{V P}$ that associate the Cartan forms of the original super $S L$ (2C) action (49) with the Cartan forms of the Volkov-Pashnev supermodel: $\omega^{\alpha}=\left.(\mathbf{a})^{1 / 2} \omega^{\alpha}\right|_{V P}, \omega^{\dot{\alpha}}=\left.\left(\mathbf{a}^{*}\right)^{1 / 2} \omega^{\dot{\alpha}}\right|_{V P}$. Then, the origin of the coefficients $\mathbf{a}$ and $\mathbf{a}^{*}$ becomes clear from the geometrical point of view.

From the first condition in (54) and the association (93) it is not difficult to see that, as in the case of the space-time cosmological constant $\Lambda: R=\frac{\Lambda}{3} e \wedge e(e \equiv$ space - time tetrad $)$, there is a cosmological term from the superspace related to the complex parameters $\mathbf{a}$ and $\mathbf{a}^{*}$ : 
$R=-\left(\mathbf{a} \omega^{\alpha} \omega_{\alpha}-\mathbf{a}^{*} \omega^{\dot{\alpha}} \omega_{\dot{\alpha}}\right)$ and it is easy to see from the minus sign in above expression, why for supersymmetric (supergravity) models it is more natural to use $S O(3,2)$ instead of SO $(4,1)$.

Note that the role of the associated spinorial action in (49) is constrained by the nature of $v \zeta_{\alpha}$ in $\mu_{p}$ as follows.

(i) If they are of the same nature of the $\omega^{\alpha}$, this term is a total derivative and has not influence onto the equations of motion, then the action proposed by Volkov and Pashnev in $[44,45]$ has the correct fermionic form.

(ii) If they are not of the same $S L(2 C)$ invariance that the $\omega^{\alpha}$, the symmetry of the original model is modified. In this direction a relativistic supersymmetric model for particles was proposed in Ref. [50] considering an N-extended Minkowsky superspace and introducing central charges to the superalgebra. Hence the underlying rigid symmetry gets enlarged to N-extended super-Poincare algebra. Considering for our case similar superextension that in Ref. [50] we can introduce the following new action

$$
\begin{aligned}
S & =-m \int_{\tau 1}^{\tau 2} d \tau \sqrt{\stackrel{\circ}{\omega}_{\mu} \omega^{\mu}+a \dot{\theta}^{\alpha} \dot{\theta}_{\alpha}-a^{*} \dot{\bar{\theta}} \dot{\dot{\theta}} \dot{\bar{\theta}}_{\dot{\alpha}}+i\left(\theta^{\alpha i} A_{i j} \dot{\theta}_{\alpha}^{j}-\bar{\theta}^{\dot{\alpha} i} A_{i j} \dot{\bar{\theta}}_{\dot{\alpha}}^{j}\right)} \\
& =\int_{\tau 1}^{\tau 2} d \tau L(x, \theta, \bar{\theta})
\end{aligned}
$$

that is the super-extended version of the superparticle model proposed in $[44,45]$ with the addition of a first-order fermionic part. The matrix tensor $A_{i j}$ introduce the symplectic structure of such manner that now $\zeta_{\alpha i} \sim A_{i j} \theta_{\alpha}^{j}$ is not covariantly constant under $d_{\omega}$. Note that the "Dirac-like" fermionic part is obviously under the square root because it is a part of the full curvature, fact that was not advertised by the authors in [50] (see also [29]) that doesn't take into account the geometrical origin of the action. An interesting point is to perform the same quantization as in the first part of the research given in [47-49] in order to obtain and compare the spectrum of physical states with the one obtained in Ref. [50]. This issue will be presented elsewhere [51].

The spontaneous symmetry breaking happens here because the parameter doesn't have any dynamics. But this doesn't happen in the nonlinear realization approach where the parameters have a particular dynamics associated with the space-time coordinates.

\section{Discussion}

Here we discuss some of the results obtained within the light of the Ref. [59] and describe their possible generalizations from the point of view of the boson-fermion symmetries as from the categories viewpoint

(i) The Darboux-Kostant theorem is fulfilled in our case showing that $\mathrm{M}$ fits the characteristic of a general even supermanifold in addition to all those the considerations given in [13,15,17,61-65]. However the extension to odd supersymplectic supermanifold is still open question

(ii) The general Rothstein theorem that we review here (also see [59] for details)is complete to describe the spacetime manifold being it with the more general symplectic even superstructure from the algebraic and geometrical viewpoint. In next work the odd part of the history must be explored. 
(iv) The possibility, following an old Dirac's conjecture, to find a discrete quaternionic structure inside the Poincare group: this fact will be give us the possibility of spacetime discretization without break Lorentz symmetries.

(v) The introduction of groupoid theoretical methods of compactification taking as group theoretical example in [71].

(vi) The relation with nonlinearly realized symmetries and geometric quantization.

With respect to [73] we introduced two geometrical models: one linear and another one quadratic in curvature. Both models are based on the $S O(2,4)$ group consequently there exists a possibility to extend the contruction to the grupoid domain. Dynamical breaking of this symmetry was considered only from the group manifold viewpoin. Because in [73] in both cases we introduced coherent states of the Klauder-Perelomov type, which as defined by the action of a group (generally a Lie group) are invariant with respect to the stability subgroup of the corresponding coset being related to the possible extension of the connection which maintains the proposed action invariant the question is if some kind of categorization of such mechanism certainly exists considering that grupiod coherent states were recently constructed [74].

From the group theoretical viewpoint [73], the linear action, unlike the cases of West or even McDowell and Mansouri [43], uses a symmetry breaking tensor that is dynamic and unrelated to a particular metric. Such a tensor depends on the introduced vectors (i.e. the coherent states) that intervene in the extension of the permissible symmetries of the original connection. Only some components of the curvature, defined by the second structure equation of Cartan, are involved in the action, leaving the remaining ones as a system of independent or ignorable equations in the final dynamics. The quadratic action, however, is independent of any additional structure or geometric artifacts and all the curvatures (e.g. all the geometrical equations for the fields) play a role in the final action (Lagrangian of the theory).

With regard to the parameters that come into play $\lambda$ and $m$ (they play the role of a cosmological constant and a mass, respectively) we saw that in the case of linear action if they are taken dependent on the coordinates and under the conditions of the action invariance, a new spontaneous compactification mechanism is defined in the subspace invariant under the stability subgroup.

Following this line of research with respect to possible physical applications, we are going to consider scenarios of the Grand Unified Theory, derivation of the symmetries of the Standard Model together with the gravitational ones. The general aim is to obtain in a precisely established way the underlying fundamental theory. The group theoretical introduction of a gauge structure and superconnections into the model, (e.g. the supergroup $S U(2 / 1)$ as the simplest case) can help to determine the fundamental structure of the underlying theory. The superconnection was introduced by Quillen in mathematics; it is a supermatrix, belonging to a given supergroup $\mathrm{S}$, valued over elements belonging to a Grassmann algebra of forms. The even part of the superconnection takes values over the gauge-potentials of the even subgroup $\mathrm{SU}(2 / 1)$ as oneforms B.dx on the base M-manifold of the bundle, realizing the "gauging" of the group G. The odd part of the supermatrix, representing the quotient $S=G=H / S$, is valued over zero-forms in that Grassmann algebra, physically interpreted as the Higgs multiplet, in a spontaneously broken G gauge theory. In quantum treatments which are set to reproduce geometrically the ghost fields and BRST equations, the Grassmann algebra is taken over the complete bundle variable. The first physical example of a superconnection preceded Quillen's theory. This was the supergroup proposal given by the authors of refs. [11] for an algebraically irreducible description of the electroweak interaction. Lacking Quillen's generalized formulation, the model appeared to suffer from spin-statistics interpre- 
tative complications for the physical fields. The structural $\mathrm{Z}$ grading of Lie superalgebras, as previously used in physics (i.e. in SUGRA, etc.), corresponds to the grading inherent in quantum statistics, i.e. to Bose-Fermi transitions, so that invariance under the supergroup represents symmetry between bosons and fermions. In the Neeman et al. proposal, however, though the superconnection itself does fit the quantum statistics ansatz, this is realized through the order of the forms in the geometrical space of the Grassmann algebra [62], rather than through the quantum statistics of the particle Hilbert space. This will be important, in particular, to solve the problem of hierarchies and fundamental constants, the masses of physical states, and their interaction that in such a case richer mathematical structures (e.g. functors, categories, etc.) can help certainly.

Acknowledgements D.J. Cirilo-Lombardo is grateful to the Bogoliubov Laboratory of Theoretical PhysicsJINR for hospitality and CONICET-ARGENTINA for .nancial support, and to Andrej B. Arbuzov for discussions and suggestions.

\section{Appendix I: Symmetry Breaking Mechanism: The $S O(4,2)$ Case}

\section{A. General Features}

(i) Let $a, b, c=1,2,3,4,5$ and $i, j, k=1,2,3,4$ (in the six-matrix representation) then the Lie algebra of $S O(2,4)$ is

$$
\begin{aligned}
i\left[J_{i j}, J_{k l}\right] & =\eta_{i k} J_{j l}+\eta_{j l} J_{i k}-\eta_{i l} J_{j k}-\eta_{j k} J_{i l}, \\
i\left[J_{5 i}, J_{j k}\right] & =\eta_{i k} J_{5 j}-\eta_{i j} J_{5 k}, \\
i\left[J_{5 i}, J_{5 j}\right] & =-J_{i j}, \\
i\left[J_{6 a}, J_{b c}\right] & =\eta_{a c} J_{6 b}-\eta_{a b} J_{6 c}, \\
i\left[J_{6 a}, J_{6 b}\right] & =-J_{a b} .
\end{aligned}
$$

(ii) Identifying the first set of commutation relations (95) as the lie algebra of the $S O(1,3)$ with generators $J_{i k}=-J_{k i}$.

(iii) The commutation relations (95) plus (96) and (97) are identified as the Lie algebra $S O(2,3)$ with the additional generators $J_{5 i}$ and $\eta_{i j}=(1,-1,-1,-1)$.

(iv) The commutation relations (95)-(99) is the Lie algebra $S O(2,4)$ written in terms of the Lorentz group $S O(1,3)$ with the additional generators $J_{5 i}, J_{6 b}$, and $J_{a b}=-J_{b a}$, where $\eta_{a b}=(1,-1,-1,-1,1)$. It follows that the embedding is given by the chain $S O(1,3) \subset S O(2,3) \subset S O(2,4)$.

From the six dimensional matrix representation we know from that parameterizing the $\operatorname{coset} \mathcal{C}=\frac{S O(2,4)}{S O(2,3)}$ and $\mathcal{P}=\frac{S O(2,3)}{S O(1,3)}$, then any element $G$ of $S O(2,4)$ is written as

$$
S O(2,4) \approx \frac{S O(2,4)}{S O(2,3)} \times \frac{S O(2,3)}{S O(1,3)} \times S O(1,3),
$$

explicitly

$$
\begin{aligned}
G & =e^{-i z^{a}(x) J_{a}} G(H) \\
& =e^{-i z^{a}(x) J_{a}} e^{-i \varepsilon^{k}(x) P_{k}} H(\Lambda) .
\end{aligned}
$$

Consequently we have $G(H): H \rightarrow G$ is an embedding of an element of $S O(2,3)$ into $S O(2,4)$ where $J_{a} \equiv \frac{1}{\lambda} J_{6 a}$ and $H(\Lambda): \Lambda \rightarrow H$ is an embedding of an element of $S O(1,3)$ 
into $S O(2,3)$ where $P_{k} \equiv \frac{1}{m} J_{5 k}$ as follows

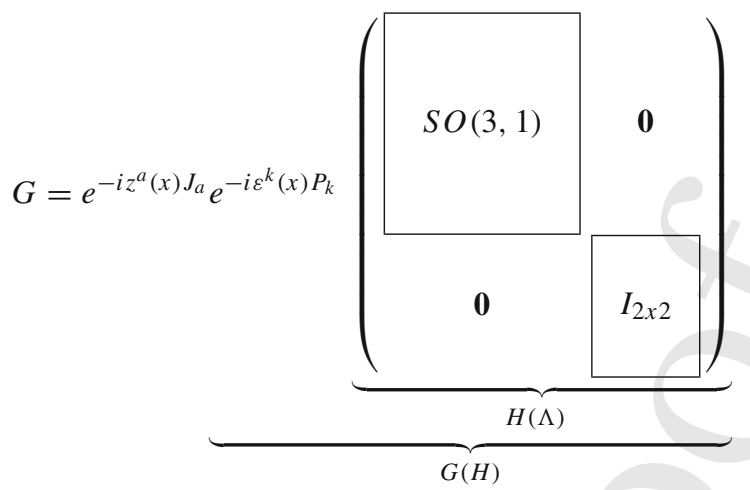

then any element $G$ of $S O(2,4)$ is written as the product of an $S O(2,4)$ boost, an $A D S$ boost, and a Lorentz rotation.

\section{Goldstone Fields and Symmetries}

(i) Our starting point is to introduce two 6-dimensional vectors $V_{1}$ and $V_{2}$ being invariant under $S O(3,1)$ in a canonical form. Explicitly

$$
\underbrace{\left(\begin{array}{c}
0 \\
0 \\
0 \\
0 \\
A \\
0
\end{array}\right)}_{V_{1}}+\underbrace{\left(\begin{array}{c}
0 \\
0 \\
0 \\
0 \\
0 \\
-B
\end{array}\right)}_{V_{2}}=\underbrace{\left(\begin{array}{c}
0 \\
0 \\
0 \\
0 \\
A \\
-B
\end{array}\right)}_{V_{0}}\} \text { invariant under } S O(3,1) .
$$

(ii) Now we take an element of $S p(2) \subset M p$ (2) embedded in the 6-dimensional matrix representation operating over $V$ as follows

$$
\mathcal{M} V \rightarrow \underbrace{\left(\begin{array}{llllll}
0 & 0 & 0 & 0 & 0 & 0 \\
0 & 0 & 0 & 0 & 0 & 0 \\
0 & 0 & 0 & 0 & 0 & 0 \\
0 & 0 & 0 & 0 & 0 & 0 \\
0 & 0 & 0 & 0 & a & b \\
0 & 0 & 0 & 0 & c & d
\end{array}\right)}_{S p(2) \subset M p(2)} \underbrace{\left(\begin{array}{c}
0 \\
0 \\
0 \\
0 \\
A \\
-B
\end{array}\right)}_{V_{0}}=\left(\begin{array}{c}
0 \\
0 \\
0 \\
0 \\
A^{\prime} \\
-B^{\prime}
\end{array}\right)=V^{\prime},
$$

where

$$
\begin{gathered}
A^{\prime}=a A-b B, \\
-B^{\prime}=c A-d B
\end{gathered}
$$

consequently we obtain a Klauder-Perelomov generalized coherent state with the fiducial vector $V_{0}$. 
(iii) The specific task to be made by the vectors is to perform the symmetry breakdown to $S O(3,1)$. Using the transformed vectors above $(S p(2) \sim M p(2) C S)$ the symmetry of $G$ can be extended to an internal symmetry as $S U(1,1)$ given by $\widetilde{G}$ below (note that $\left.|\lambda|^{2}-|\mu|^{2}=1\right)$ :
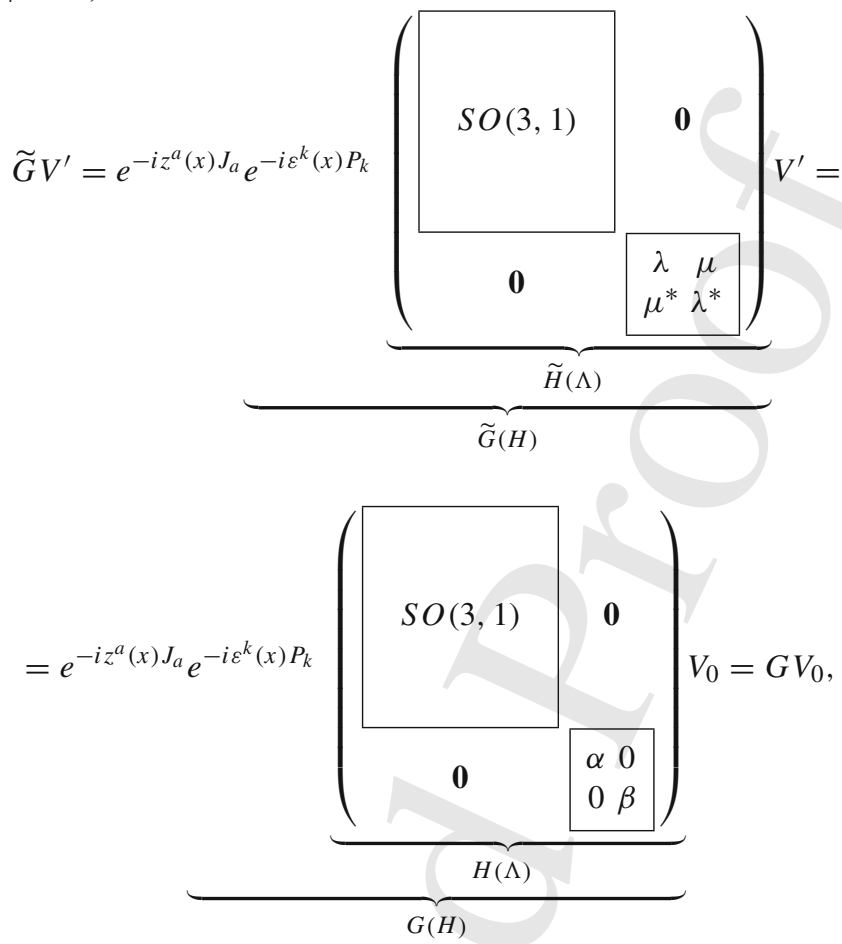

$$
\mathcal{M}=\left(\begin{array}{cccccc}
0 & 0 & 0 & 0 & 0 & 0 \\
0 & 0 & 0 & 0 & 0 & 0 \\
0 & 0 & 0 & 0 & 0 & 0 \\
0 & 0 & 0 & 0 & 0 & 0 \\
0 & 0 & 0 & 0 & \lambda^{*} \alpha & -\mu \beta \\
0 & 0 & 0 & 0 & -\mu^{*} \alpha & \lambda \beta
\end{array}\right)
$$

and if we also ask for $\operatorname{Det} \mathcal{M}=1$ then $\alpha \beta=1$, e.g. the additional phase: it will bring us the $10^{t h}$ Goldstone field. The other nine are given by $z^{a}(x)$ and $\varepsilon^{k}(x)(a, b, c=$ $1,2,3,4,5$ and $i, j, k=1,2,3,4)$ coming from the parameterization of the cosets $\mathcal{C}=\frac{S O(2,4)}{S O(2,3)}$ and $\mathcal{P}=\frac{S O(2,3)}{S O(1,3)}$. (e.g. geometrically $\left.A d S_{4} \times S_{3}\right)$.

\section{References}

1. Isaev, A.P.: Quantum group covariant noncommutative geometry. J. Math. Phys. 35, 6784 (1994). arXiv:hep-th/9402060

2. Aschieri, P., Castellani, L., Isaev, A.P.: Discretized Yang-Mills and Born-Infeld actions on finite group geometries. Int. J. Mod. Phys. A 18, 3555 (2003). arXiv:hep-th/0201223

3. Blagojevic, M.: Gravitation and Gauge Symmetries, p. 522. IOP, Bristol (2002)

4. Hayashi, K., Shirafuji, T.: Gravity from poincare gauge theory of the fundamental particles. 7. The axial vector model. Prog. Theor. Phys. 66, 2258 (1981)

\section{Springer}


5. Borisov, A.B.: The unitary representations of the general covariant group algebra. J. Phys. A 11, 1057 (1978)

6. Utiyama, R.: Invariant theoretical interpretation of interaction. Phys. Rev. 101, 1597 (1956)

7. Capozziello, S., De Laurentis, M.: Extended theories of gravity. Phys. Rep. 509, 167 (2011). [arXiv:1108.6266 [gr-qc]]

8. Hehl, F.W., McCrea, J.D., Mielke, E.W., Ne'eman, Y.: Metric affine gauge theory of gravity: field equations, Noether identities, world spinors, and breaking of dilation invariance. Phys. Rep. 258, 1 (1995). [gr-qc/9402012]

9. Ivanenko, D., Sardanashvily, G.: The gauge treatment of gravity. Phys. Rep. 94, 1 (1983)

10. Obukhov, Y.N.: Poincare gauge gravity: selected topics. Int. J. Geom. Methods Mod. Phys. 3, 95 (2006). [gr-qc/0601090]

11. Ne'eman, Y., Regge, T.: Gauge theory of gravity and supergravity on a group manifold. Riv. Nuovo Cim. 1N5, 1 (1978)

12. Gotzes, S., Hirshfeld, A.C.: A geometric formulation of the $\operatorname{SO}(3,2)$ theory of gravity. Ann. Phys. 203, 410 (1990)

13. Shirafuji, T., Suzuki, M.: Gauge theory of gravitation: a unified formulation of poincare and anti-de sitter gauge theories. Prog. Theor. Phys. 80, 711 (1988)

14. Ivanov, E.A., Niederle, J.: Gauge formulation of gravitation theories. 1. The poincare, de sitter and conformal cases. Phys. Rev. D 25, 976 (1982)

15. Ivanov, E.A., Niederle, J.: Gauge formulation of gravitation theories. 2. The special conformal case. Phys. Rev. D 25, 988 (1982)

16. Leclerc, M.: The Higgs sector of gravitational gauge theories. Ann. Phys. 321, 708 (2006). [gr-qc/0502005]

17. Stelle, K.S., West, P.C.: Spontaneously broken de sitter symmetry and the gravitational holonomy group. Phys. Rev. D 21, 1466 (1980)

18. Tseytlin, A.A.: On the poincare and de sitter gauge theories of gravity with propagating torsion. Phys. Rev. D 26, 3327 (1982)

19. Lord, E.A., Goswami, P.: Gauge theory of a group of diffeomorphisms. 1. General principles. J. Math. Phys. 27, 2415 (1986)

20. Lord, E.A.: Gauge theory of a group of diffeomorphisms. 2. The conformal and de sitter groups. J. Math. Phys. 27, 3051 (1986)

21. Greenberg, M.: Lectures on Algebraic Topology. W.A. Benjamin Inc., Menlo Park (1971)

22. Kobayashi, S., Nomizu, K.: Foundations of Differential Geometry. Wiley, New York (1963)

23. Sardanashvily, G.: Classical gauge gravitation theory. Int. J. Geom. Methods Mod. Phys. 8, 1869 (2011). [arXiv:1110.1176 [math-ph]]

24. Giachetta, G., Mangiarotti, L., Sardanashvily, G.: Advanced Classical Field Theory. World Scientific, Singapore (2009)

25. Kirsch, I.: A Higgs mechanism for gravity. Phys. Rev. D 72, 024001 (2005). arXiv:hep-th/0503024

26. Keyl, M.: About the geometric structure of symmetry breaking. J. Math. Phys. 32, 1065 (1991)

27. Nikolova, L., Rizov, V.A.: Geometrical approach to the reduction of gauge theories with spontaneously broken symmetry. Rep. Math. Phys. 20, 287 (1984)

28. Sardanashvily, A.: On the geometry of spontaneous symmetry breaking. J. Math. Phys. 33, 1546 (1992)

29. Sardanashvily, G.: Geometry of classical Higgs fields. Int. J. Geom. Methods Mod. Phys. 3, 139 (2006). arXiv:hep-th/0510168

30. Sardanashvily, G.: Mathematical models of spontaneous symmetry breaking. arXiv:0802.2382 [math-ph]

31. Sardanashvily, G.: Classical Higgs fields. Theor. Math. Phys. 181, 1598 (2014). [arXiv:1602.03818 [math$\mathrm{ph}]]$

32. Trautman, A.: Differential Geometry For Physicists, p. 145. Bibliopolis, Naples (1984)

33. Lawson, H.B., Michelsohn, M.L.: Spin Geometry. Princeton University Press, Princeton (1989)

34. Sardanashvily, G.: Gravity as a goldstone field in the lorentz gauge theory. Phys. Lett. A 75, 257 (1980)

35. Sardanashvily, G.A., Zakharov, O.: Gauge Gravitation Theory, p. 122. World Scientific, Singapore (1992)

36. Hawking, S.W., Ellis, G.F.R.: The Large Scale Structure of Space-Time, p. 404. Cambridge University Press, Cambridge (1973)

37. Sardanashvily, G.: What are the poincare gauge fields? Czech. J. Phys. B 33, 610 (1983)

38. Volkov, D.V., Soroka, V.A.: Higgs effect for goldstone particles with spin 1/2. JETP Lett. 18, 312 (1973)

39. Volkov, D.V., Soroka, V.A.: Higgs effect for goldstone particles with spin 1/2. Pisma Zh. Eksp. Teor. Fiz. 18, $529(1973)$

40. Akulov, V.P., Volkov, D.V., Soroka, V.A.: Gauge fields on superspaces with different holonomy groups. JETP Lett. 22, 187 (1975)

41. Akulov, V.P., Volkov, D.V., Soroka, V.A.: Gauge fields on superspaces with different holonomy groups. Pisma Zh. Eksp. Teor. Fiz. 22, 396 (1975) 
42. Nath, P., Arnowitt, R.L.: Generalized supergauge symmetry as a new framework for unified gauge theories. Phys. Lett. 56B, 177 (1975)

43. MacDowell, S.W., Mansouri, F.: Unified Geometric Theory of Gravity and Supergravity. Phys. Rev. Lett. 38, 739 (1977) Erratum: [Phys. Rev. Lett. 38, 1376 (1977)]

44. Volkov, D.V., Pashnev, A.I.: Supersymmetric lagrangian for particles in proper time. Theor. Math. Phys. 44, 770 (1980)

45. Volkov, D.V., Pashnev, A.I.: Supersymmetric Lagrangian for particles in proper time. Teor. Mat. Fiz. 44, 321 (1980)

46. Inonu, E., Wigner, E.P.: On the contraction of groups and their represenations. Proc. Nat. Acad. Sci. 39, 510 (1953)

47. Cirilo-Lombardo, D.J.: Non-compact groups, coherent states, relativistic wave equations and the Harmonic Oscillator. Found. Phys. 37, 919 (2007)

48. Cirilo-Lombardo, D.J.: Non-compact groups, coherent states, relativistic wave equations and the Harmonic Oscillator. Found. Phys. 37, 1149 (2007)

49. Cirilo-Lombardo, D.J.: Non-compact groups, coherent states, relativistic wave equations and the Harmonic Oscillator. Found. Phys. 38, 99 (2008). arXiv:hep-th/0701195

50. de Azcarraga, J.A., Lukierski, J.: Supersymmetric particle model with additional bosonic coordinates. Z. Phys. C 30, 221 (1986)

51. Cirilo-Lombardo, D.J., Arbuzov, A.: Electroweak dynamical symmetries beyond the SM and coherent states. Work in progress

52. Ogievetsky, V.I.: Infinite-dimensional algebra of general covariance group as the closure of finitedimensional algebras of conformal and linear groups. Lett. Nuovo Cim. 8, 988 (1973)

53. Volkov, D.V., Akulov, V.P.: Is the neutrino a goldstone particle? Phys. Lett. 46B, 109 (1973)

54. Capozziello, S., Cirilo-Lombardo, D.J., De Laurentis, M.: The affine structure of gravitational theories: symplectic groups and geometry. Int. J. Geom. Methods Mod. Phys. 11(10), 1450081 (2014)

55. Borisov, A.B., Ogievetsky, V.I.: Theory of dynamical affine and conformal symmetries as gravity theory. Theor. Math. Phys. 21, 1179 (1975)

56. Borisov, A.B., Ogievetsky, V.I.: Theory of dynamical affine and conformal symmetries as gravity theory. Teor. Mat. Fiz. 21, 329 (1974)

57. Cirilo-Lombardo, D.J.: Non-compact groups, coherent states, relativistic wave equations and the harmonic osscillator II: physical and geometrical considerations. Found. Phys. 39, 373-396 (2009)

58. Cirilo-Lombardo, D.J.: The geometrical properties of Riemannian superspaces, exact solutions and the mechanism of localization. Phys. Lett. B 661, 186-191 (2008)

59. Cirilo-Lombardo, D.J.: Algebraic structures, physics and geometry from a unified field theoretical framework. Int. J. Theor. Phys. 54(10), 3713-3727 (2015)

60. Ambrose, W., Singer, I.M.: A theorem on holonomy. Trans. Am. Math. Soc. 75(3), 428-443 (1953)

61. Kostant, B.: Graded manifolds, graded Lie theory and pre-quantization. In: Bleuler, K., Reetz, A. (eds.) Differential Geometrical Methods in Mathematical Physics: Proceedings of the Symposium Held at the University of Bonn, July 1-4, 1975. Lecture Notes in Mathematics, vol. 570, pp. 177-306. Springer, Berlin (1977)

62. Rothstein, M.: The structure of supersymplectic supermanifolds. In: Bartocci, C., Bruzzo, U., Cianci, R. (eds.) Differential Geometric Methods in Theoretical Physics. Lecture Notes in Physics, vol. 375. Springer, Berlin (1991)

63. Bartocci, C., Bruzzo, U., Hernandez Ruiperez, D.: The Geometry of Supermanifolds. Kluwer, Dordrecht (1991)

64. Winnberg, J.O.: Superfields as an extension of the spin representation of the orthogonal group. J. Math. Phys. 18, 625 (1977)

65. Pavsic, M.: Spin Gauge Theory of Gravity in Clifford Space. J. Phys. Conf. Ser. 33, 422-427 (2006)

66. Pavsic, M.: A theory of quantized fields based on orthogonal and symplectic Clifford Algebras. Adv. Appl. Clifford Algebras 22, 449-481 (2012)

67. Albert, A.A.: Structure of Algebras. American Mathematical Society, Providence, RI (1961)

68. Salingaros, N.A., Wene, G.P.: The Clifford algebra of differential forms. Acta Appl. Math. 4(27), 1-292 (1985)

69. Pavsic, M.: On the unification of interactions by Clifford algebra. Adv. Appl. Clifford Algebras 20, 781-801 (2010)

70. Pavsic, M.: Space inversion of spinors revisited: a possible explanation of chiral behavior in weak interactions. Phys. Lett. B692, 212-217 (2010)

71. Cirilo-Lombardo, D.J.: Geometrical properties of Riemannian superspaces, observables and physical states. Eur. Phys. J. C72, 2079 (2012) 
72. Mickelsson, J.: Boundary currents and hamiltonian quantization of fermions in background fields. Phys. Lett. B 456, 124-128 (1999)

73. Cirilo-Lombardo, D.J., Arbuzov, A.: Dynamical symmetries, coherent states and nonlinear realizations: the SO(2,4) case. Int. J. Geom. Methods Mod. Phys. 15(01), 1850005 (2017)

74. Agyo, S., Lei, C., Vourdas, A.: The groupoid of bifractional transformations. ArXiv:1706.03557 